\title{
LEF1 is preferentially expressed in the tubal-peritoneal junctions and is a reliable marker of tubal intraepithelial lesions
}

\author{
Elisa Schmoeckel ${ }^{1}$, Ashley A Odai-Afotey ${ }^{2}$, Michael Schleißheimer ${ }^{1}$, Miriam Rottmann ${ }^{3}$, \\ Andrea Flesken-Nikitin ${ }^{2}$, Lora H Ellenson ${ }^{4}$, Thomas Kirchner ${ }^{1}$, Doris Mayr ${ }^{1}$ and \\ Alexander Yu Nikitin ${ }^{2}$ \\ ${ }^{1}$ Institute of Pathology, Ludwig Maximilians University, Munich, Germany; ${ }^{2}$ Department of Biomedical \\ Sciences and Cornell Stem Cell Program, Cornell University, Ithaca, NY, USA; ${ }^{3}$ Institute of Medical \\ Information Processing, Biometry und Epidemiology, Ludwig Maximilians University, Munich, Germany and \\ ${ }^{4}$ Department of Pathology and Laboratory Medicine, Weill Cornell Medicine, New York, NY, USA
}

\begin{abstract}
Recently it has been reported that serous tubal intraepithelial carcinoma (STIC), the likely precursor of ovarian/ extra-uterine high-grade serous carcinoma, are frequently located in the vicinity of tubal-peritoneal junctions, consistent with the cancer-prone features of many epithelial transitional regions. To test if p53 (aka TP53)signatures and secretory cell outgrowths (SCOUTs) also localize to tubal-peritoneal junctions, we examined these lesions in the fallopian tubes of patients undergoing salpingo-oophorectomy for sporadic high-grade serous carcinomas or as a prophylactic procedure for carriers of familial BRCA1 or 2 mutations. STICs were located closest to the tubal-peritoneal junctions with an average distance of $1.31 \mathrm{~mm}$, while SCOUTs were not detected in the fimbriated end of the fallopian tube. As many epithelial transitional regions contain stem cells, we also determined the expression of stem cell markers in the normal fallopian tube, tubal intraepithelial lesions and high-grade serous carcinomas. Of those, LEF1 was consistently expressed in the tubal-peritoneal junctions and all lesions, independent of p53 status. All SCOUTs demonstrated strong nuclear expression of $\beta$-catenin consistent with the LEF1 participation in the canonical WNT pathway. However, $\beta$-catenin was preferentially located in the cytoplasm of cells comprising STICs and p53 signatures, suggesting WNT-independent function of LEF1 in those lesions. Both frequency of LEF1 expression and $\beta$-catenin nuclear expression correlated with the worst 5-year patient survival, supporting important role of both proteins in high-grade serous carcinoma. Taken together, our findings suggest the existence of stem cell niche within the tubal-peritoneal junctions. Furthermore, they support the notion that the pathogenesis of SCOUTs is distinct from that of STICs and p53 signatures. The location and discrete patterns of LEF1 and $\beta$-catenin expression may serve as highly sensitive and reliable ancillary markers for the detection and differential diagnosis of tubal intraepithelial lesions.
\end{abstract}

Modern Pathology (2017) 30, 1241-1250; doi:10.1038/modpathol.2017.53; published online 30 June 2017

Ovarian carcinoma is the most lethal gynecological malignancy and is fifth among all cancer related deaths of women in the US. ${ }^{1}$ During the past decade a number of studies have shown that about half of the most malignant ovarian/extra-uterine cancers, high-grade serous carcinomas, may arise from the

Correspondence: Dr E Schmoeckel or Dr D Mayr, Institute of Pathology, Ludwig-Maximilians-University Munich, Thalkirchnerstraße 36, 80337 Munich, Germany or Dr AY Nikitin, Department of Biomedical Sciences and Cornell Stem Cell Program, 618 Tower Road T2 014A VRT, Cornell University, Ithaca, NY, USA.

E-mail: elisa.schmoeckel@med.uni-muenchen.de or doris.mayr@med. uni-muenchen.de or an58@cornell.edu

Received 20 January 2017; revised 10 April 2017; accepted 19 April 2017; published online 30 June 2017 fallopian tube epithelium. ${ }^{2}$ In this situation, neoplastic tubal lesions lead to metastatic disease with the main manifestation in the ovary. This possibility is supported by the finding of dysplastic lesions in prophylactically removed fallopian tubes from highrisk carriers of BRCA1/2 germline mutations, ${ }^{3}$ detection of identical p53 mutations in early tubal lesions and high-grade serous carcinomas of the same patients, ${ }^{4,5}$ and experimental demonstration of high-grade serous carcinoma induction by conditional mutations in the secretory (PAX8 positive) cells of mouse tubal epithelium. ${ }^{6}$ Considering that ovarian cancer can be successfully treated if diagnosed at an early stage, with $90 \%$ of such patients 
having over a 5-year survival, its early detection is of particular importance.

Early dysplastic lesions associated with high-grade serous carcinoma include p53 (aka TP53) signatures, serous tubal intraepithelial carcinomas (STICs) and potentially secretory cell outgrowth (SCOUTs). p53 signatures are characterized by areas of a single layer of consecutive PAX8-positive secretory cells that contain aberrant p53 expression, commonly associated with p53 mutations, but lack cellular atypia and show a low proliferative index. ${ }^{4}$ STICs also show aberrant p53 expression and express PAX8, but are characterized by dysplastic epithelial cells with the loss of cell polarity and high proliferative index. ${ }^{7-10} \mathrm{~A}$ large fraction of STICs $(30-50 \%)$ completely lack p53 expression, largely due to p53 null mutation in the p53 gene, and their detection by immunohistochemistry may be more challenging. ${ }^{4,5,9}$ SCOUTs consist of a row of at least 30 almost exclusively PAX8-positive secretory epithelial cells with a pseudostratified benign appearance and low proliferative index. ${ }^{11-13}$

p53 signatures and STICs are preferentially located in the distal, fimbriated region of the fallopian tube, ${ }^{4}$ whereas SCOUTs are reported to be evenly distributed between fimbriated and more proximal areas of the fallopian tube. ${ }^{13}$ However, it remains elusive if SCOUTs, p53 signatures and STICs represent independent or continuous stages of neoplastic progression. ${ }^{11,13,14}$

A recent study demonstrated that the majority STICs are located in the vicinity of the tubalperitoneal junction. ${ }^{15}$ The tubal-peritoneal junctions connects the columnar epithelium of the fimbriated end of the fallopian tube to the flat mesothelial layer of the peritoneum. The finding of STICs near the tubal-peritoneal junctions is consistent with previous observations that transitional epithelial zones, such as the corneal limbus and squamo-columnar junctions in the uterine cervix, gastro-esophageal, and ano-rectal areas are frequently cancer prone. ${ }^{16-18}$ Furthermore, it may offer an equivalent to the recently discovered cancer-prone stem cell niche in the mouse hilum area, which connects the ovarian surface epithelium, tubal epithelium, and mesothelium. ${ }^{19}$ The location of p53 signatures and distal SCOUTs with respect to tubalperitoneal junctions remains unknown. Such information could provide an important clue to their role as precursor lesions to STICs.

One consistent marker of hilum stem cells is lymphoid enhancer-binding factor-1 (LEF1). LEF1 is a transcription factor that interacts with $\beta$-catenin in the nucleus as a component of canonical WNT signaling. ${ }^{20}$ LEF1 expression has been reported in human STICs and SCOUTs. ${ }^{21}$ However, the frequency and extent of this expression were not described. Furthermore, expression of LEF1 in normal tubal-peritoneal junctions and p53 signatures remains unknown.

In this study we compared the distance from the tubal-peritoneal junctions to STICs, p53 signatures and SCOUTs in the fallopian tubes of patients undergoing salpingo-oophorectomy for sporadic high-grade serous carcinomas or as prophylactic procedures for carriers of familial BRCA1 or 2 mutations. Furthermore, we have determined expression of LEF1 and other stem cell markers in the tubalperitoneal junctions, tubal intraepithelial lesions and high-grade serous carcinomas. We have also compared survival of patients with tumors containing high and low number of LEF1-expressing cells.

\section{Materials and methods}

\section{Study Groups and Clinical Data}

Material in these studies was submitted to the Institute of Pathology, Ludwig Maximilians University, Munich, Germany between 2009 and 2014. Cases included fallopian tubes from 42 patients with sporadic highgrade serous carcinoma without BRCA1/2 germline mutation (further denoted as 'high-grade serous carcinoma group'), from 44 patients tested positive for $B R C A 1$ or 2 germline mutations (further denoted as 'BRCA group'), and 31 control cases.

In the high-grade serous carcinoma group the status of somatic BRCA mutation of tumor was unknown. In order to avoid that a bulky tumor obscured the potential tubal intraepithelial lesions only cases with complete right and left tube and presentable fimbria were included. Cases with advanced tumor mass in the tube and invasion of one or both tubes were excluded. Patient's age ranged from 42 to 85 years, with an average of 65 years. 37 patients were of advanced tumor-stage (FIGO $\geq$ IIIA). Two patients presented with a FIGO stage I (one of IA and one of IC). Three patients were of FIGO stage II. A representative paraffin block of the invasive cancer was obtained for immunohistochemical stainings.

In the BRCA group 1 patient presented with an early invasive high-grade serous carcinoma of the fallopian tube (FIGO IC). All other cases were from prophylactic salpingo-oophorectomy and encompassed 28 cases of BRCA1- and 16 cases of BRCA2mutation. Patients' age ranged from 33 to 79 years, with an average of 52 years.

Control cases of fallopian tubes were from 31 patients who underwent hysterectomy for leiomyoma of the uteri or carcinoma of the cervix. Patients' age ranged from 40 to 74 years, with an average of 56 years.

Location and size of STICs and their distance to tubal-peritoneal junctions were additionally evaluated in 10 cases (5 high-grade serous carcinoma, 2 BRCA and 3 without either high-grade serous carcinoma or BRCA) from Weill Cornell Medicine, New York, New York.

\section{Histotechnology and Immunohistochemistry}

The fallopian tubes were fixed in buffered formalin and prepared for paraffin embedding according to 
the SEE-FIM-protocol. ${ }^{8}$ Serial $4 \mu \mathrm{m}$ sections were prepared from each paraffin block and mounted on SuperFrost Plus microscope slides (Menzel Gläser, Braunschweig, Germany). After deparaffinization and rehydration step-wise slides were stained with hematoxylin and eosin, while parallel slides were subjected immunohistochemical stainings for p53, Ki67, PAX2, PAX8, CD44, CD49f, CD133, LEF1, and $\beta$-catenin (Supplementary Table 1). For $\beta$-catenin two different antibodies were used. All cases were first stained automatically. Cases of unsecure nuclear reaction were stained additionally by manual staining.

Stainings for $\beta$-catenin, CD133, Ki67, p53, and PAX8 were subjected to heat-induced epitope unmasking with pressure cooker heating followed by staining using a Ventana Benchmark XT autostainer (Ventana Medical Systems, Oro Valley, AZ, USA) with the XT UltraView diaminobenzidine kit (Vector Laboratories, Burlingame, CA, USA) and hematoxylin counterstaning (Vector Laboratories, Burlingame, CA, USA).

For CD44, LEF1, $\beta$-catenin, CD49f, and PAX2 manual staining slides were exposed to heat pretreatment, followed by incubation with primary antibodies for $1 \mathrm{~h}$ at room temperature, secondary anti-species antibodies (Vectastain, ABC kit universal, PK 6200, Vector Laboratories, Burlingame, CA, USA for LEF1, $\beta$-catenin BD and CD44; ImmPRESS reagent kit, anti-rabbit IgG, MP -7401, Vector Laboratories, Burlingame, CA, USA for CD49f and PAX2) and detection with ABC Elite kit (Vector Laboratories, Burlingame, CA, USA). The DAB+ system (Dako, Hamburg, Germany) was used as chromogen and the slides were then counterstained with hematoxylin (Vector Laboratories, Burlingame, CA, USA). System controls were included.

\section{Analysis of Lesions}

STIC is defined by a proliferation of dysplastic epithelial cells with loss of cell polarity and absence of ciliated cells. PAX8 is expressed continuously. STIC is marked by increased proliferation (Ki67 $>10 \%$ of cells) and aberrant status of p53 immunostaining, which includes either strong diffuse staining of p53 or complete absence of p53 staining, as compared with weak nuclear staining in neighboring stromal cells. ${ }^{7-9,22-25}$

p53 signatures are morphologically inconspicuous and consists of at least 12 consecutive PAX8-positive secretory cells, that are p53 positive, but lack any cellular atypia and show a low proliferative index $(\mathrm{Ki} 67<10 \%)^{4,7,23,24}$

SCOUTs consist of at least 30 almost exclusively secretory PAX8-positive epithelial cells with a pseudostratified appearance. Immunohistochemistry for p53 is negative and the proliferative index is low (Ki67 $<10 \%)^{11-13}$ The cells of these lesions usually contain wild-type p53 manifested as weak nuclear staining. Such staining is also typically observed in stromal cells.

All lesions were independently analyzed by several pathologists (ES, LHE, DM, and AYN). All histological sections were digitized at giga-pixel resolution, 24-bit color, and $40 \times$ magnification using Aperio ScanScope CS2 (Leica Biosystems, Buffalo Grove, IL, USA). Image analysis was performed using ImageJ software (National Institute of Health, Bethesda, MD, USA). Size of STICs, p53 signatures and SCOUTs was determined in parallel sections followed by 3D reconstruction of lesions. The distance to the tubal-peritoneal junctions was measured for all STICs, p53 signatures and SCOUTs located in the same tissue section as the tubalperitoneal junctions.

For the immunohistochemical analysis of LEF1 expression in the tubal-peritoneal junctions 15 mesothelial cells and 60 tubal epithelial cells were evaluated. Together a total length of 400-550 $\mu \mathrm{m}$ (in most cases $475 \mu \mathrm{m}$ ) was counted for each junction. The number of junctions found per analyzed case ranged from 1 to 5 and the average expression was calculated for each case. The referring distant regions were chosen and defined based on them having overall representative staining composition of the distal tubal epithelium in each case. The average of two distant regions, each consisting of 60 cells was calculated.

Expression of LEF1 in STICs, p53 signatures, SCOUTs and high-grade serous carcinoma was quantified as the percentage of positive stained cells in each lesion using ImageJ cell counter in digital images. Due to injured tubal mucosa or missing tubal-peritoneal junctions on parallel slides used for the immunohistochemical stainings, subsets of 19 high-grade serous carcinoma cases, 20 BRCA cases, and 12 controls were used for detailed analysis of immunohistochemical stainings. Among those, 21 STICs, 16 p53 signatures, and 15 SCOUTs were stained for LEF1, $\beta$-catenin, CD44, and CD133. Highgrade serous carcinoma were considered to be positive for LEF1 and nuclear $\beta$-catenin in cases with over $30 \%$ stained neoplastic cells.

\section{Statistical Analysis}

Statistical analysis was performed using SPSS version 19.0 (PASW Statistics; SPSS Inc., IBM, Chicago, IL, USA), GraphPad Prism version 6 (GraphPad, La Jolla, USA) and MedCalc version 17 (Ostend, Belgium). An average percent positivity and standard deviation was taken for each region for each independent uterine tube case. Average percent positivity was used in all statistical analysis. Unpaired $t$-tests with Welch's correction (Gaussian distribution) or Mann-Whitney tests (nonparametric test) were used to determine if within a given region differences in means between markers were statistically significant. Overall survival was analyzed 
using Kaplan-Meier method with log rank test and Cox proportional hazards regression. $P$-values of $<0.05$ were considered statistically significant.

\section{Results}

\section{Incidence and Size of Tubal Intraepithelial Lesions}

Among 19 STIC cases in the high-grade serous carcinoma group (Table 1), 7 cases presented with a small solid tumor mass in the ovary $(\leq 3.5 \mathrm{~cm}$ in diameter) and an ipsilateral manifestation of STIC. The remaining 12 cases presented with a larger solid and cystic tumor mass in the ovaries. In 9 out of 19 STIC cases in the high-grade serous carcinoma group a microscopic invasive tumor in the fimbria was bordering the STIC. In the BRCA group one case included an early high-grade serous carcinoma of the fallopian tube (FIGO IC) and a contralateral STIC as well as SCOUT on the side of the invasive cancer.

10 out of $19(52.63 \%)$ STICs completely lacked p53 immunostaining, as typical for p53 null mutations, in the high-grade serous carcinoma group. In all cases the p53 immunostaining status (aberrant accumulation vs complete lack of staining) in STICs was identical in the high-grade serous carcinoma of the same patient. In the BRCA group 2 out of 4 (50\%) STICs had complete lack of p53 staining. One case of the BRCA group contained a STIC with complete lack of p53 staining and a p53 signature in the same tube.

The average size of STICs $(2.73 \mathrm{~mm})$ was significantly larger than p53 signatures $(1.12 \mathrm{~mm}, P=0.0004)$ and SCOUTs $(1.04 \mathrm{~mm}, P=0.0001$; Table 2). Sizes of

Table 1 Distribution of tubal intraepithelial lesions in study groups

\begin{tabular}{lccc}
\hline Lesion/group & HGSC, $\mathrm{n}=42^{\mathrm{a}}$ & BRCA, $\mathrm{n}=44^{\mathrm{a}, \mathrm{b}}$ & Control, $\mathrm{n}=31^{\mathrm{a}}$ \\
\hline STIC & $19(45 \%)$ & $4(9 \%)$ & 0 \\
p53 signature & $10(24 \%)$ & $6(14 \%)$ & $5(16 \%)$ \\
SCOUT & $11(26 \%)$ & $4(9 \%)$ & $3(10 \%)$ \\
No lesions & $14(33 \%)$ & $34(77 \%)$ & $26(84 \%)$ \\
\hline
\end{tabular}

${ }^{\text {a }}$ Some cases include several types of lesions. ${ }^{\mathrm{b}} \mathrm{BRCA}$ group includes BRCA1 $(n=29)$ and BRCA2 $(n=15)$ cases with STIC/p53 signature/ SCOUT distribution $3 / 5 / 3$ and $1 / 1 / 1$, respectively.
SCOUTs and p53 signatures were not significantly different $(P=0.6974)$.

\section{Distance from Tubal Intraepithelial Lesions to Tubal- Peritoneal Junctions}

Consistent with previous reports, ${ }^{2,4}$ the majority of STICs $(85 \%)$ and p53 signatures $(62 \%)$ were located in the fimbriated region (Table 2; Figure 1a). Furthermore, no STICs were located more proximally than the infundibulum $(15 \%)$, while $19 \%$ of p53 signatures were found in the ampulla region. Importantly, the majority of SCOUTs were observed in the infundibulum (65\%), followed by the ampulla (26\%).

Among lesions located in the fimbriated region, STICs tend to be closer to the tubal-peritoneal junctions (mean distance $1.34 \mathrm{~mm}$ ) than p53 signatures $(1.96 \mathrm{~mm})$; however this difference is not statistically significant $(P=0.1217)$. In three cases, STICs were located directly at the tubal-peritoneal junctions and were flanked by the mesothelial layer at the one side and columnar tubal epithelium at the other (Figure 1b).

\section{Increased Frequency of LEF1-Expressing Cells in the Tubal-Peritoneal Junctions and all Tubal Intraepithelial Lesions}

Since epithelial transitional regions may contain stem cell niches, we have evaluated a panel of markers characteristic for stem/progenitor cell, such as LEF1, CD44, CD49f, CD133, and $\beta$-catenin in the morphologically normal fallopian tube epithelium at the tubal-peritoneal junctions and in the proximal tubal epithelium of the same section at distance of $4 \mathrm{~mm}$. Among those markers the frequency of LEF1 positive cells was significantly higher in the area of tubal-peritoneal junctions. LEF1-positive cells were also more frequent in morphologically normal epithelium of high-grade serous carcinoma and BRCA groups (Figure 2). There were no preferential distribution of CD44, CD49f, and $\beta$-catenin stained cells, while CD133 was mainly detected in the proximal region of the fallopian tube.

The average number of LEF1-positive cells was significantly higher in STIC, p53 signature, and SCOUT compared with the bordering normal tubal epithelium (Figure 3). Importantly, STICs completely

Table 2 Size, location and distance to the TPJ among tubal intraepithelial lesions

Location

\begin{tabular}{|c|c|c|c|c|c|c|}
\hline \multirow{2}{*}{ Lesion/group } & \multirow{2}{*}{ Size, $m m \pm$ s.d. } & & \multirow{2}{*}{ Distance to $T P J, m m \pm s . d^{a}$} \\
\hline & & Fimbriae & Infundibulum & Ampulla & Isthmus & \\
\hline STIC, $n=33^{\mathrm{b}}$ & $2.73 \pm 1.55$ & $28(85 \%)$ & $5(15 \%)$ & $0(0 \%)$ & $0(0 \%)$ & $1.34 \pm 1.15$ \\
\hline p53 signature, $n=16$ & $1.12 \pm 0.90^{\mathrm{C}}$ & $10(62 \%)$ & $3(19 \%)$ & $3(19 \%)$ & $0(0 \%)$ & $1.96 \pm 1.55$ \\
\hline SCOUT, $n=15$ & $1.04 \pm 0.33^{\mathrm{d}}$ & $1(4 \%)$ & $15(65 \%)$ & $6(26 \%)$ & $1(4 \%)$ & NA \\
\hline
\end{tabular}

${ }^{\mathrm{a}}$ For lesions located in the fimbria. ${ }^{\mathrm{b}}$ Includes 10 cases from Weill Cornell Medicine, New York. ${ }^{\mathrm{c}} P=0.0004$ vs STIC. ${ }^{\mathrm{d}} P=0.0001$ vs STIC. 
a
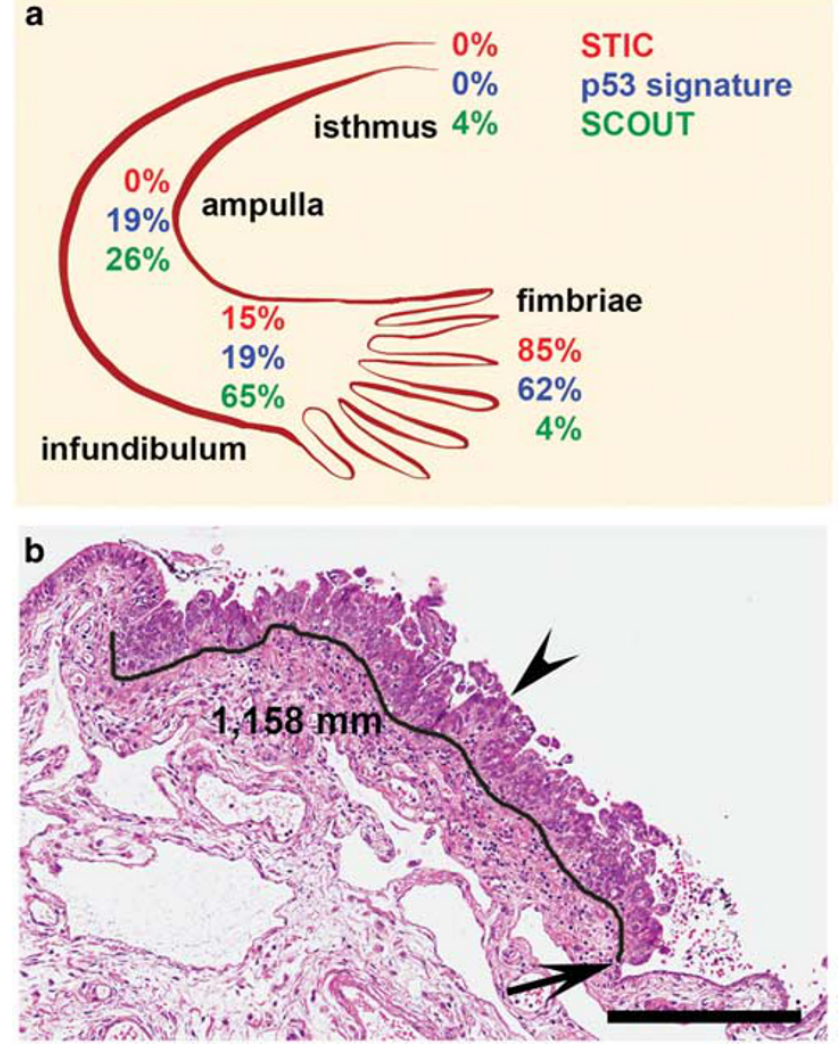

Figure 1 Lesions in the fallopian tube. (a) Distribution of tubal lesions. (b) STIC (arrowhead) at the tubal-peritoneal junction (arrow), flanked by the mesothelium and the tubal epithelium at the right and the left sides, respectively. Line shows the length of STIC. Hematoxylin and eosin. Scale bar, $300 \mu \mathrm{m}$.

lacking p53 expression also expressed LEF1, thereby simplifying detection of such lesions. (Figures 3c and d).

\section{Divergent $\beta$-catenin Cellular Location Differentiates STICs, Signatures, and High-Grade Serous Carcinoma from SCOUTs}

In all SCOUTs the expression of LEF1 was associated with a marked nuclear expression of $\beta$-catenin (Figure 3). STICs and p53 signatures showed only minimal nuclear expression of $\beta$-catenin in single cells, whereas the majority of cells in these lesions presented with a distinct membranous expression of $\beta$-catenin.

To establish if divergent expression of LEF1 and $\beta$-catenin is also present in advanced malignancies, immunostaining was additionally performed in 35 cases of sporadic high-grade serous carcinoma (Figure 4). Nuclear expression was similar to that of intraepithelial lesions, but the frequency of LEF1ranged from 0 to $75 \%$ (mean \pm s.d., $18 \pm 16.7 \%$ ). Similar to STICs and p53 signatures, $\beta$-catenin showed a distinct membranous expression in the cancers with only minimal nuclear expression in the majority of cases. However, focal nuclear expression was detected in all high-grade serous carcinoma cases studied, ranging from 2 to $55 \%$. As compared with LEF1, expression of $\beta$-catenin was observed more uniformly in all high-grade serous carcinoma cases. Patient's survival correlated significantly with frequency of LEF1 and nuclear $\beta$-catenin positive cells in the cancer (Figure 4).

\section{Discussion}

Recent studies of risk-reducing salpingo-oophorectomies in women with BRCA1/2 germline mutations showed that STICs are identified in $2-12 \%$ of the patients. ${ }^{2,14,26}$ In context of high-grade serous carcinoma the incidence of STIC ranges rather up to 19$60 \% .{ }^{15,27-29}$ Our studies have found the frequency of STICs to be $9 \%$ and $45 \%$ in the above groups, respectively, thereby confirming the earlier observations. It should be noted that all of these studies cannot exclude that some STICs may represent metastatic implants from the associated high-grade serous carcinoma. ${ }^{30,31}$ Due to its proximity to the ovary, the location of the majority of STICs in the fimbriae may be the preferential site of implantation from extra-tubal carcinomas. In such a scenario, it would be more likely to observe random distribution of tumor implants within the tubal fimbria. However, consistent with the recent study by Seidman, ${ }^{15}$ we have observed preferential location of STICs at or in the immediate vicinity to the tubal-peritoneal junctions. Furthermore, we have observed similar distribution of STICs in the BRCA1/2 cases, where no overt tumor was observed. This is consistent with the notion that a large fraction of lesions described as STICs are of primary, not metastatic implantation, origin.

Consistent with previous observations, ${ }^{11,13,14}$ we have shown that STICs and p53 signatures are preferentially located within the tubal fimbria, while SCOUTs are detected in the more proximal regions of the tube. Both p53 signatures and SCOUTs have approximately the same size (about $1.1 \mathrm{~mm}$ on average), while STICs are 2.5 times larger $(2.73 \mathrm{~mm})$. All STICs and p53 signatures but not SCOUTs contain aberrant p53 immunostaining. In high-grade serous carcinoma cases, the type of p53 immunostaining, either aberrant accumulation or aberrant lack of staining, were identical to the carcinoma of the same patient. Further supporting the common pathogenesis of STICs and p53 signatures, in contrast to that of SCOUTs, only SCOUTs have marked nuclear location of $\beta$-catenin, a key component of the canonical WNT pathway. STICs, p53 signatures as well as high-grade serous carcinomas were characterized by preferential membrane and cytoplasmic staining with only minimal nuclear location of $\beta$-catenin. In sum, based on the distinct location and different patterns of p53 and $\beta$-catenin expression in STICs/p53 signatures, p53 signatures 
but not SCOUTs are likely to represent the steps in the continuous progression towards STICs and overt high-grade serous carcinoma. Consistent with this
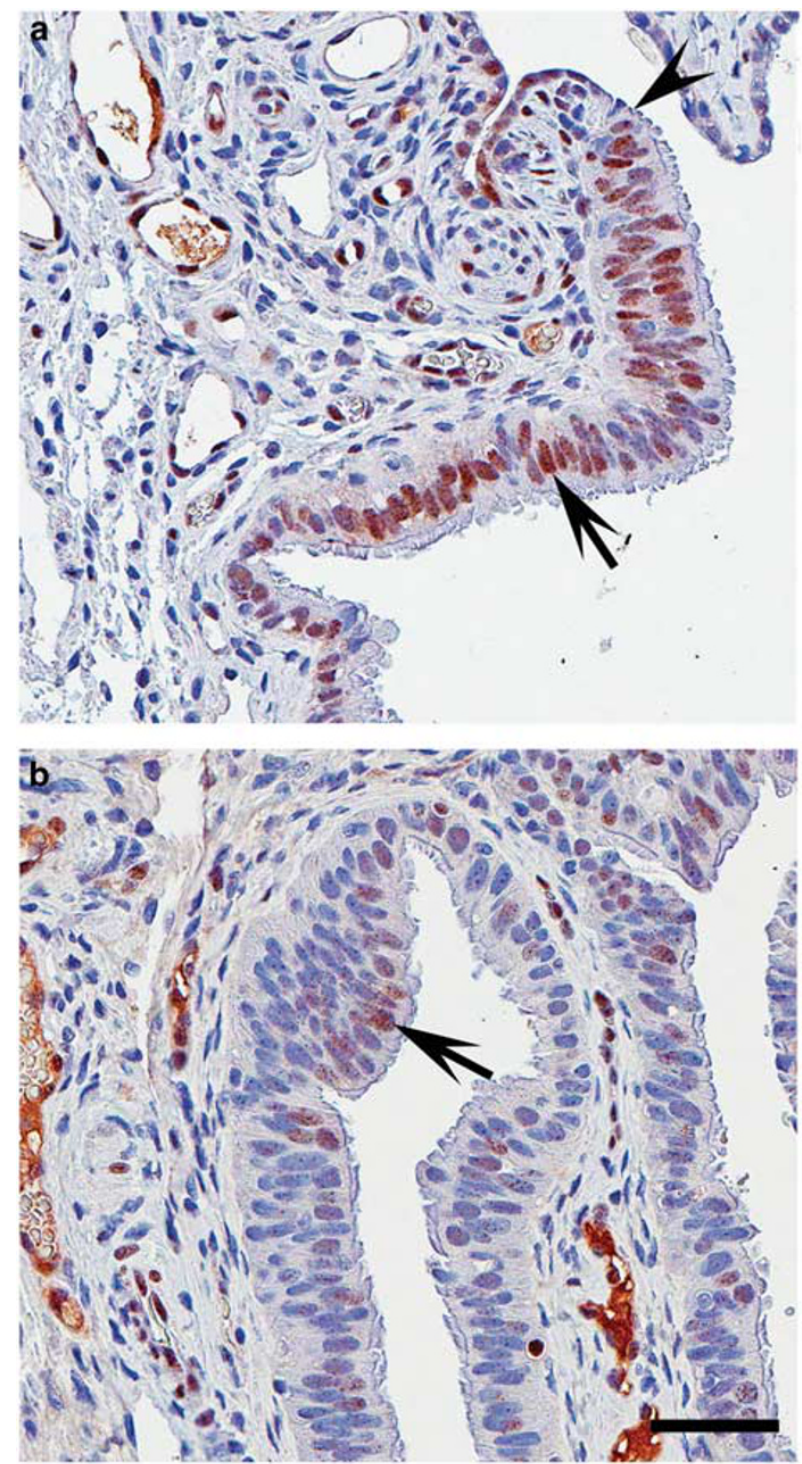

C

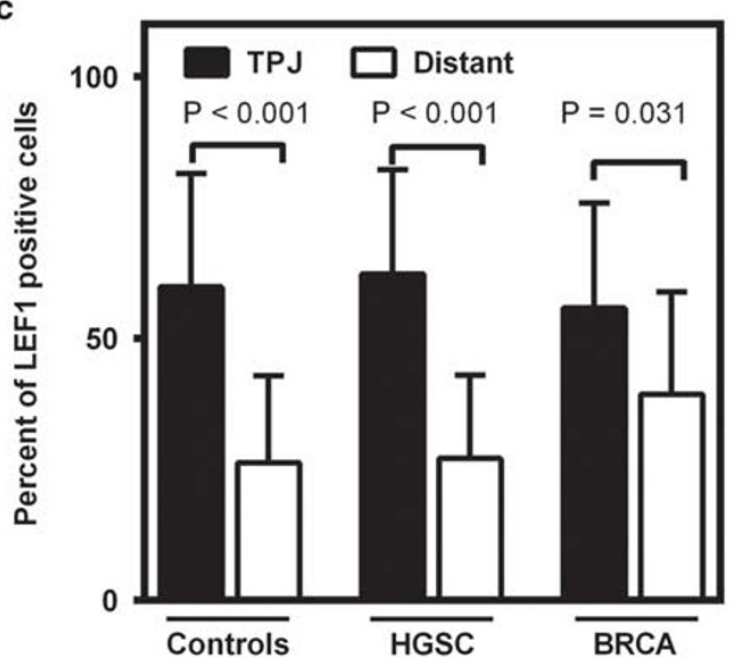

notion, the frequency of p53 signatures is two times higher than that of STICs in fallopian tubes from women in the BRCA group (14\% vs 9\%). However, in high-grade serous carcinoma group, these frequencies are inverse (24\% vs $45 \%)$ suggesting that either all STIC's arise from p53 signatures or that some STICs may in fact represent implants of highgrade serous carcinoma. Consistent with this possibility, according to recent next-generation sequencing, a STIC represented a micro-metastasis in $25 \%$ of cases. ${ }^{31,32}$ As in our study SCOUTs are defined by an accumulation of at least 30 secretory cells, we cannot rule out that some smaller groups of secretory cells might exist in the distal tube and progress to p53 signatures and/or STICs. More extensive results based on targeting tubal epithelium stem cells and their lineages in combination with single cell genome sequencing will better inform us in future.

Previously, we have reported the existence of a cancer-prone stem cell niche in the junction/transitional zone between ovarian surface epithelium, tubal epithelium and mesothelium located within the mouse hilum of the ovary. ${ }^{19}$ In humans, the same hilum-based transitional zone is not maintained. However, the tubal-peritoneal junctions of the tubal fimbria may represent a counterpart to the mouse junction zone. Consistent with the possibility of stem cell niche located in the tubal-peritoneal junctions we have observed expression of LEF1 in that area. LEF1 is expressed in a number of normal stem cells, such as hematopoietic ${ }^{33}$ and ovarian surface epithelium stem cells. ${ }^{19}$ Moreover, deregulated LEF1 expression has been implicated in leukemic transformation. ${ }^{33}$ Further studies testing functional properties of tubal-peritoneal junction cells should specifically evaluate the key parameters of stem cells, such as their ability to self-renew and to give rise to more differentiated progeny. These studies will also test if putative stem cells previously identified in the distal end of the fallopian tube ${ }^{34}$ are identical or similar to LEF1 positive cells found in the tubal-peritoneal junctions area. We have noted that other tested stem/progenitor cell markers did not have preferential association with cells located near the tubal-peritoneal junctions. Some of these markers, such as CD44 and CD49f (aka integrin $\alpha 6$ ) have been reported to be expressed in putative stem cells of the human tubal epithelium. ${ }^{34}$ Thus it is possible that the tubal epithelium may have several

Figure 2 LEF1 expression in the morphologically normal tubal epithelium. (a) LEF1 expression in a large fraction of cells (arrow) in the tubal epithelium near the tubal-peritoneal junctions region (arrowhead) of the control group. (b) LEF1 in few cells (arrow) of the tubal epithelium of the proximal region of the control group. ABC Elite method, hematoxylin counterstaining. Scale bar, $50 \mu \mathrm{m}$. (c) Quantitative analysis of LEF1 expression in control $(n=12)$, high-grade serous carcinoma $(n=19)$ and BRCA $(n=16)$ groups. Error bars denote s.d. 
a

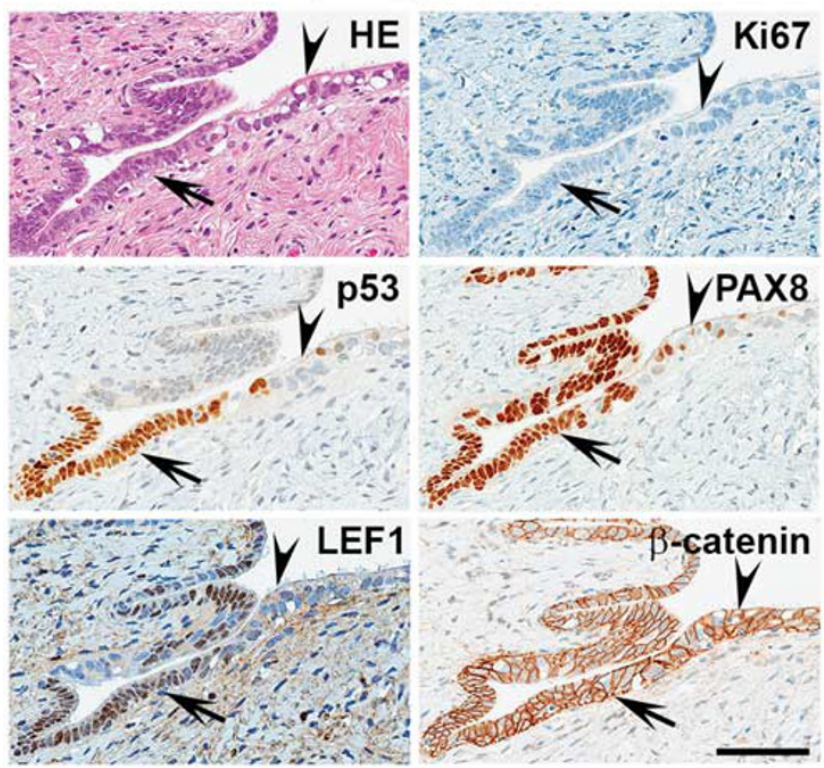

C
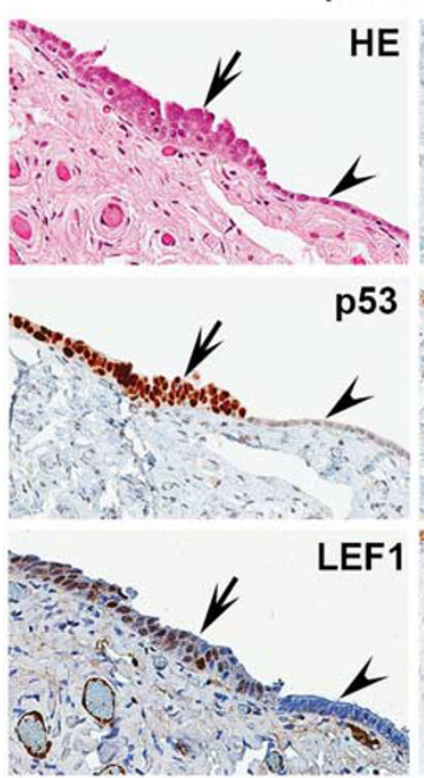

b

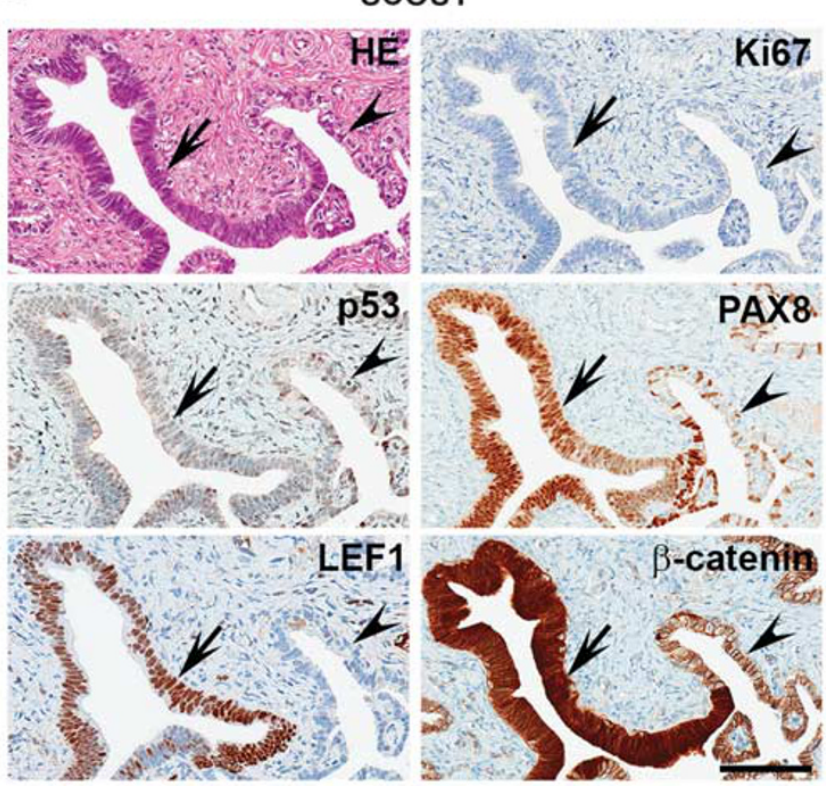

d

p53 cl STIC
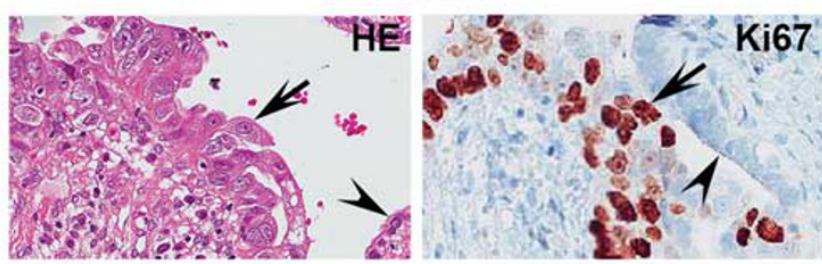

p53
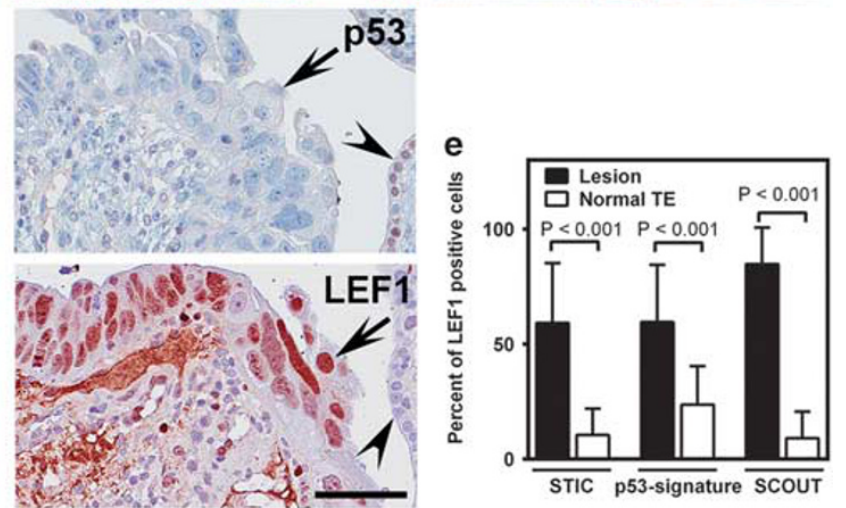

Figure 3 Immunohistochemical characterization of tubal lesions. (a-d) Expression of p53, LEF1, Ki67, PAX8, and $\beta$-catenin in the altered (arrow) and morphologically normal (arrowhead) epithelium in p53 signature (a), SCOUT (b), and STIC cases with p53 aberrant accumulation (aa), (c) and p53 complete lack (cl) of staining. (d) Note mainly membrane staining of $\beta$-catenin in p53 signatures and STICs. ABC Elite method, hematoxylin counterstaining, HE, hematoxylin and eosin. Scale bars, $70 \mu \mathrm{m}$ for all images. (e) Quantitative analysis of LEF1 expression in STIC $(n=21)$, p53 signature $(n=16)$, SCOUT $(n=15)$ and adjacent morphologically normal tubal epithelium (TE). Error bars denote s.d.

pools of stem cells. At the same time, such markers are not always entirely specific and may label nonstem cells in some tissues.

LEF1 is frequently found to be an important component of WNT pathway. ${ }^{20}$ However, according to our studies, LEF1 expression in p53 signatures, SCOUTs and high-grade serous carcinomas does not correlate with the nuclear location of $\beta$-catenin, a property of active WNT signaling. Thus it is likely that the LEF1 function in these lesions are WNT pathway independent. WNT-independent functions of LEF1 were previously described in granulocyte progenitor cells ${ }^{35}$ and murine T-cell lymphomas. ${ }^{36}$ At the same time, we observed a strong coincidence of nuclear accumulation of $\beta$-catenin and LEF1 expression in SCOUTs, supporting a distinct pathogenesis of SCOUTs, as compared with other tubal lesions. We have also shown that both high LEF1 

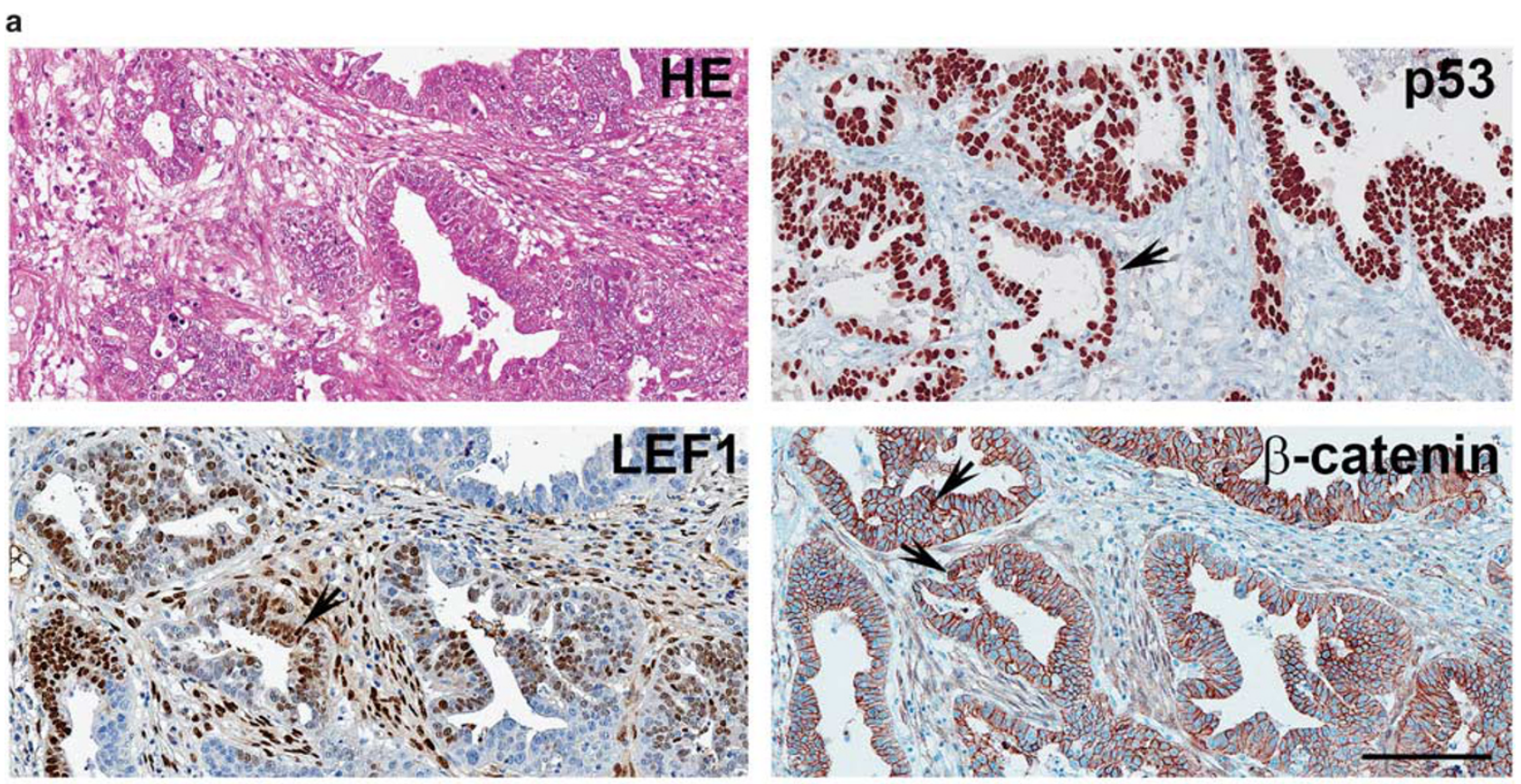

b
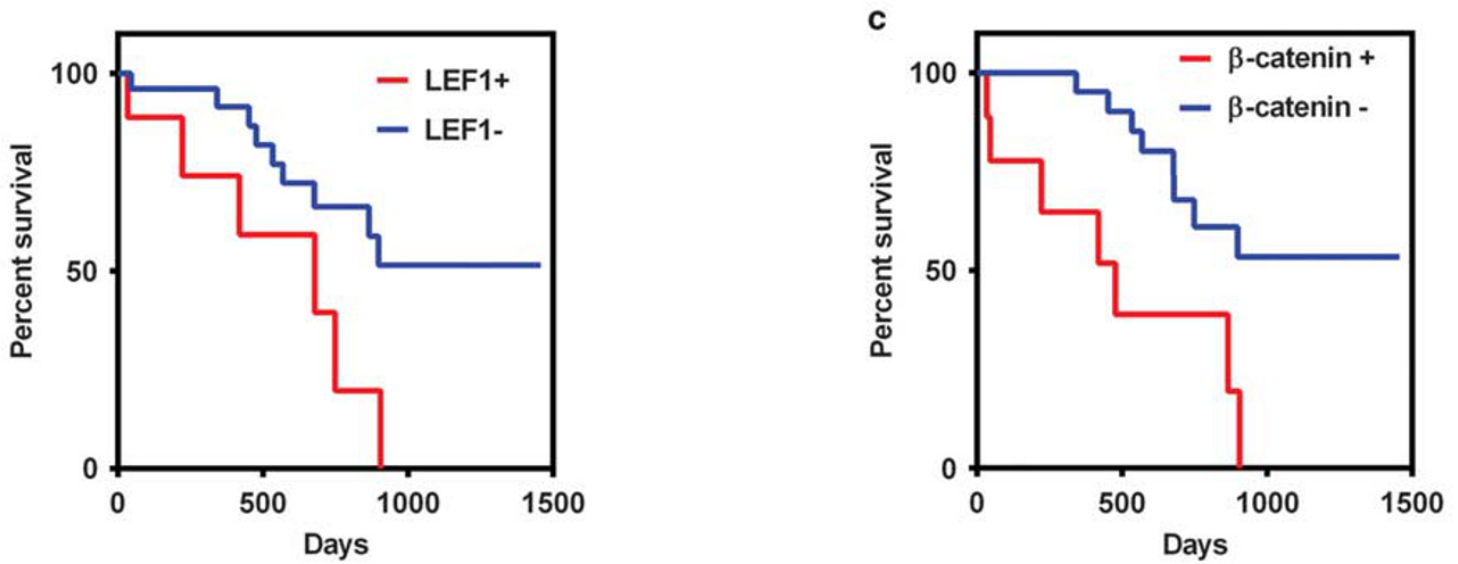

Figure 4 LEF1 and $\beta$-catenin in high-grade serous carcinoma. (a) Expression of p53, LEF1 and $\beta$-catenin. Arrows indicated nuclear staining. Note mainly membrane staining of $\beta$-catenin in neoplastic cells. Parallel sections of the same block. ABC Elite method, hematoxylin counterstaining, HE, hematoxylin and eosin. Scale bar, $150 \mu \mathrm{m}$ for all images. (b,c) Survival of patients with high-grade serous carcinoma stratified according to LEF1 (b) and nuclear $\beta$-catenin (c) expression. Kaplan-Meier survival analysis, $n=35, P=0.0306$ (a) and $P=0.0029$ (b). Cox proportional hazards regression analysis $P=0.0394$ (a) and $P=0.0060$ (b).

expression and nuclear location of $\beta$-catenin correlate with decreased survival of high-grade serous carcinoma patients. Further studies should elucidate specific mechanisms by which LEF1 regulates the function of stem and neoplastic cells in tubalperitoneal junctions, tubal lesions and high-grade serous carcinoma. They also should address the extent to which both WNT pathway dependent and independent functions contribute to the high-grade serous carcinoma pathogenesis.

Our study has shown that LEF1 expression together with strong nuclear location of $\beta$-catenin is indicative of SCOUTs, while LEF1 expression together with largely membranous and cytoplasmic $\beta$-catenin is typical for p53 signatures and STICs. Thus LEF1 may be used as an auxiliary marker for detection of tubal lesions, particularly in conjunction with $\beta$-catenin detection. This could be a particularly useful tool for the detection of TP53 with aberrant lack of immunostaining in STICs and p53 signatures. It has been previously reported that high LEF1 expression correlates with poor prognosis of acral melanoma $^{37}$ and colorectal cancer, ${ }^{38}$ B-precursor acute lymphoblastic leukemia ${ }^{39}$ and in chronic lymphocytic leukemia. ${ }^{40,41}$ Our studies indicate that LEF1 expression may be also a useful prognostic marker in ovarian cancer. As both LEF1 and strong nuclear location of $\beta$-catenin correlate with a worse prognosis, further studies on a larger cohort of patients are needed to establish if correlation between these parameters has additional prognostic value. 
In sum, our findings support the existence of cancer-prone stem cell niche within the tubalperitoneal junctions. They also suggest that the pathogenesis of STICs and p53 signatures is distinct from that of SCOUTs. Furthermore, detection of LEF1 expression, especially in combination with $\beta$-catenin intracellular distribution, may be used as a highly sensitive and reliable tool for the diagnosis of tubal intraepithelial lesions, particularly well-suited for detection of STICs with aberrant lack of p53 immunostaining.

\section{Acknowledgments}

We thank Andrea Sendelhofert, Anja Heier, Jessica Kövi, Tera Kent and Christopher L. Pelletier for their excellent technical assistance. This study was supported by the Wilhelm Sander-foundation to ES and DM, and by the grants from the New York Stem Cell Program (C028125, C029155, and T028095), the National Institutes of Health/The National Cancer Institute (CA182413) and Ovarian Cancer Research Fund (327516) to AYN.

\section{Disclosure/conflict of interest}

The authors declare no conflict of interest.

\section{References}

1 Siegel RL, Miller KD, Jemal A. Cancer Statistics, 2017. CA Cancer J Clin 2017;67:7-30.

2 Perets R, Drapkin R. It's totally tubular...riding the new wave of ovarian. Cancer Res 2016;76:10-17.

3 Piek JM, van Diest PJ, Zweemer RP, et al. Dysplastic changes in prophylactically removed fallopian tubes of women predisposed to developing ovarian cancer. J Pathol 2001;195:451-456.

4 Lee Y, Miron A, Drapkin R, et al. A candidate precursor to serous carcinoma that originates in the distal fallopian tube. J Pathol 2007;211:26-35.

5 Kuhn E, Kurman RJ, Vang R, et al. TP53 mutations in serous tubal intraepithelial carcinoma and concurrent pelvic high-grade serous carcinoma-evidence supporting the clonal relationship of the two lesions. J Pathol 2012;226:421-426.

6 Perets R, Wyant GA, Muto KW, et al. Transformation of the fallopian tube secretory epithelium leads to highgrade serous ovarian cancer in Brca;Tp53;Pten models. Cancer Cell 2013;24:751-765.

7 Carcangiu ML, Radice P, Manoukian S, et al. Atypical epithelial proliferation in fallopian tubes in prophylactic salpingo-oophorectomy specimens from BRCA1 and BRCA2 germline mutation carriers. Int J Gynecol Pathol 2004;23:35-40.

8 Medeiros F, Muto MG, Lee Y, et al. The tubal fimbria is a preferred site for early adenocarcinoma in women with familial ovarian cancer syndrome. Am J Surg Pathol 2006;30:230-236.

9 Novak M, Lester J, Karst AM, et al. Stathmin 1 and p16 (INK4A) are sensitive adjunct biomarkers for serous tubal intraepithelial carcinoma. Gynecol Oncol 2015; 139:104-111.

10 Meserve EE, Brouwer J, Crum CP. Serous tubal intraepithelial neoplasia: the concept and its application. Mod Pathol 2017;30:710-721.

11 Chen EY, Mehra K, Mehrad M, et al. Secretory cell outgrowth, PAX2 and serous carcinogenesis in the Fallopian tube. J Pathol 2010;222:110-116.

12 Wang Y, Li L, Wang Y, Tang SN, Zheng W. Fallopian tube secretory cell expansion: a sensitive biomarker for ovarian serous carcinogenesis. Am J Transl Res 2015;7: 2082-2090.

13 Quick CM, Ning G, Bijron J, et al. PAX2-null secretory cell outgrowths in the oviduct and their relationship to pelvic serous cancer. Mod Pathol 2012;25:449-455.

14 Cass I, Walts AE, Barbuto D, Lester J, Karlan B. A cautious view of putative precursors of serous carcinomas in the fallopian tubes of BRCA mutation carriers. Gynecol Oncol 2014;134:492-497.

15 Seidman JD. Serous tubal intraepithelial carcinoma localizes to the tubal-peritoneal junction: a pivotal clue to the site of origin of extrauterine high-grade serous carcinoma (ovarian cancer). Int J Gynecol Pathol 2015;34:112-120.

16 McNairn AJ, Guasch G. Epithelial transition zones: merging microenvironments, niches, and cellular transformation. Eur J Dermatol 2011;21:21-28.

17 Herfs M, Yamamoto Y, Laury A, et al. A discrete population of squamocolumnar junction cells implicated in the pathogenesis of cervical cancer. Proc Natl Acad Sci USA 2012;109:10516-10521.

18 Flesken-Nikitin A, Odai-Afotey AA, Nikitin AY. Role of the stem cell niche in the pathogenesis of epithelial ovarian cancers. Mol Cell Oncol 2014;1:1-7.

19 Flesken-Nikitin A, Hwang CI, Cheng CY, et al. Ovarian surface epithelium at the junction area contains a cancerprone stem cell niche. Nature 2013;495:241-245.

20 Clevers H, Loh KM, Nusse R. Stem cell signaling. An integral program for tissue renewal and regeneration: Wnt signaling and stem cell control. Science 2014;346: 1248012.

21 Ning G, Bijron JG, Yamamoto Y, et al. The PAX2-null immunophenotype defines multiple lineages with common expression signatures in benign and neoplastic oviductal epithelium. J Pathol 2014;234: 478-487.

22 Tang S, Onuma K, Deb P, et al. Frequency of serous tubal intraepithelial carcinoma in various gynecologic malignancies: a study of 300 consecutive cases. Int J Gynecol Pathol 2012;31:103-110.

23 Gross AL, Kurman RJ, Vang R, Shih Ie M, Visvanathan K. Precursor lesions of high-grade serous ovarian carcinoma: morphological and molecular characteristics. J Oncol 2010;2010:126295.

24 Jarboe E, Folkins A, Nucci MR, et al. Serous carcinogenesis in the fallopian tube: a descriptive classification. Int J Gynecol Pathol 2008;27:1-9.

25 Visvanathan K, Vang R, Shaw P, et al. Diagnosis of serous tubal intraepithelial carcinoma based on morphologic and immunohistochemical features: a reproducibility study. Am J Surg Pathol 2011;35:1766-1775.

26 Callahan MJ, Crum CP, Medeiros F, et al. Primary fallopian tube malignancies in BRCA-positive women undergoing surgery for ovarian cancer risk reduction. J Clin Oncol 2007;25:3985-3990.

27 Kindelberger DW, Lee Y, Miron A, et al. Intraepithelial carcinoma of the fimbria and pelvic serous carcinoma: 
Evidence for a causal relationship. Am J Surg Pathol 2007;31:161-169.

28 Crum CP, Herfs M, Ning G, et al. Through the glass darkly: intraepithelial neoplasia, top-down differentiation, and the road to ovarian cancer. J Pathol 2013;231: 402-412.

29 Przybycin CG, Kurman RJ, Ronnett BM, Shih IeM, Vang R. Are all pelvic (nonuterine) serous carcinomas of tubal origin? Am J Surg Pathol 2010;34:1407-1416.

30 Stewart CJ. Fallopian tube intraluminal tumor spread. Am J Surg Pathol 2014;38:578-579.

31 Eckert MA, Pan S, Hernandez KM, et al. Genomics of ovarian cancer progression reveals diverse metastatic trajectories including intraepithelial metastasis to the fallopian tube. Cancer Discov 2016;6:1342-1351.

32 McDaniel AS, Stall JN, Hovelson DH, et al. Nextgeneration sequencing of tubal intraepithelial carcinomas. JAMA Oncol 2015;1:1128-1132.

33 Petropoulos K, Arseni N, Schessl C, et al. A novel role for Lef-1, a central transcription mediator of Wnt signaling, in leukemogenesis. J Exp Med 2008;205: $515-522$.

34 Paik DY, Janzen DM, Schafenacker AM, et al. Stem-like epithelial cells are concentrated in the distal end of the fallopian tube: a site for injury and serous cancer initiation. Stem Cells 2012;30:2487-2497.

35 Skokowa J, Cario G, Uenalan M, et al. LEF-1 is crucial for neutrophil granulocytopoiesis and its expression is severely reduced in congenital neutropenia. Nat Med 2006;12:1191-1197.

36 Spaulding C, Reschly EJ, Zagort DE, et al. Notch1 co-opts lymphoid enhancer factor 1 for survival of murine T-cell lymphomas. Blood 2007;110:2650-2658.

$37 \mathrm{Xu} \mathrm{S}$, Yang Z, Zhang J, et al. Increased levels of beta-catenin, LEF-1, and HPA-1 correlate with poor prognosis for acral melanoma with negative BRAF and NRAS mutation in BRAF exons 11 and 15 and NRAS exons 1 and 2. DNA Cell Biol 2015;34:69-77.

38 Wang WJ, Yao Y, Jiang LL, et al. Increased LEF1 expression and decreased Notch2 expression are strong predictors of poor outcomes in colorectal cancer patients. Dis Markers 2013;35:395-405.

39 Kuhnl A, Gokbuget N, Kaiser M, et al. Overexpression of LEF1 predicts unfavorable outcome in adult patients with B-precursor acute lymphoblastic leukemia. Blood 2011;118:6362-6367.

40 Erdfelder F, Hertweck M, Filipovich A, Uhrmacher S, Kreuzer KA. High lymphoid enhancer-binding factor-1 expression is associated with disease progression and poor prognosis in chronic lymphocytic leukemia. Hematol Rep 2010;2:e3.

41 Gutierrez A Jr., Tschumper RC, Wu X, et al. LEF-1 is a prosurvival factor in chronic lymphocytic leukemia and is expressed in the preleukemic state of monoclonal B-cell lymphocytosis. Blood 2010;116: 2975-2983.

Supplementary Information accompanies the paper on Modern Pathology website (http://www.nature.com/ modpathol) 